\title{
Efecto de la Aplicación del Bioabono en el Rendimiento de Cultivos de Vigna ${ }^{1}$
}

\author{
Nelson Roberto Flores Blandón ${ }^{2}$
}

\section{COMPENDIO}

Al utilizar técnicas como la digestión anaeróbica del estiércol oovino puede producirse, en la propia finca, materiales orgánicos para aportarlos al suelo, con un costo relativamente bajo.

De la digestión anaeróbica se obtienen productos como el biogás y el bioaoono, este último posee excelentes propiedades físico-químicas que lo convierten en una buena fuente de materia orgánica, con características fertilizantes y mejoradoras del suelo.

Con el objetivo principal de evaluar el efecto que sobre el rendimiento de vigna, Vigna unguiculata, tienen las aplicaciones del bioabono; se estableció en un segundo año, un ensayo de campo en la Escuela Nacional de Agricultura, a una altitud de $460 \mathrm{msnm}$, un. precipitación media anual de $1800 \mathrm{~mm}$ y una temperatura media anual de $23.8^{\circ} \mathrm{C}$. El suelo en estudio pertenece al grupo Regosol aluvial de textura franco-arenosa.

Se evaluaron cuatro tratamientos de fertilización de los cuales tres niveles fueron de aplicación de bioabono: 40,80 y $120 \mathrm{~m}^{3} / \mathrm{ha}$ y lino de aplicación de urea además de un testigo absoluto. El bioabono fue aplicado al suelo ocho días antes de siembra y la urea se aplicó en bandas ocho días después de la siembra.

Los rendimientos en peso fresco fueron similares estadísticamente en los tratamientos con bioaoono y la aplicación de Urea y se observó una leve atendencia creciente en los rendimientos obtenidos en este segundo año con respecto a los obtenidos en el primer año, esto hace suponer dque los efectos del hioaoooosobre esta variable serían mejores a través del tiempo.

Palabras claves adicionales: Fertilización, aoono orgánico, Vigna unguiculata.

\section{INTRODUCCIÓN}

La materia orgánica presente en el suelo tiene efectos múltiples sobre las propiedades físicas, químicas y microbiológicas del mismo. Cuando estos efectos se combinan sobre dichas propiedades, como resultado se obtiene un efecto integrado en el rendimiento de los cultivos (Charreau, 1976).

La materia orgánica para abastecer al suelo proviene de diversas fuentes vegetales o animales lo que influye en su calidad; aunque en todo caso las técnicas para su obtención y adición al suelo deben ser relativamente sencillas, además de económicas para que la rentabilidad de su utilización sea aceptable.

Una de estas técnicas es la biodigestión anaeróbica de los estiércoles animales y/o residuos vegetales en la cual

\begin{abstract}
3
Organic materials as a soil amendment may be chellply prod used in farmer' s fields via anaerobic digestion of beef manure. From the anaerobic digestion, one obtains gas and manure, the latter having excellent physical and chemical properties which makes it a good source of organic matter.

The objectives of this work were to evaluate the effects of beef manure applications on Vigna (Vigna unguiculata) yields Experiments were conducted for two years at the National School of Agriculture, El Salvador, 460 meters aoove sea level, $1800 \mathrm{~mm}$ annual precipitation, and a mean temperature of $23.8^{\circ} \mathrm{C}$. The soil in this study was an alluvial saody loam Regosol.

A total of five treatments were evaluated that included three rates of applied beef manure $\left(40,80,120 \mathrm{~m}^{3} / \mathrm{ha}\right)$, an inorganic fertilizer check $(0.39$ aud $0.20 \mathrm{~kg} / 20 \mathrm{~m}^{2}$ of ammonium sulfate and urea respectively) and an absolute check (no $\mathrm{N}$ applied). The beef manure was applied to the soil eightdays before planting. The urea fraction of the inorganic fertilizer check was banded 8 days after planting.

Fresh weight yields were statistically similar between manure and the inorganic fertilizer check in the tirst year, however, a slight tendency for higher yields was observed for the second year. This suggests that applications of manure can improve Vigna fresh weight yields over time.
\end{abstract}

además de producirse gas combustible (biogás: 70\% metllilo, $30 \%$ dióxido de carbono) se produce un residuo conocido como bioabono.

El biocabono es la parte sólida no digerida presente en el efluente de un biodigestor. Se caracteriza por ser rico en materia orgánica y nutrimentos, usualmente contiene un $2 \%$ de nitrógeno, $1.5 \%$ de fósforo, $1.8 \%$ de potasio y otros elementos. Dichas características lo convierten en un material orgánico ideal para aplicarse en los suelos agrícolas, especialmente en aquellos donde la materia orgánica es escasa.

\footnotetext{
1 Trabajo presentado en la XXXVI Reunión Anual del PCCMCA, San Salvador, El Salvador, marzo 1990

${ }^{2}$ Tec. log. Agrícola. Técnico del Departamento de Suelos. CENTA.

${ }^{3}$ El abstraet es traducción del compendio.

Publicado en Agronomía Mesoamericana, Vol. 2 (1991).
} 
Un experimento de campo se realizó durante dos años con el objeto principal de evaluar el efecto del bioabono sobre el rendimiento de Vigna unguiculata. Se presentan los resultados obtenidos en dos ciclos de aplicación (19881989).

\section{REVISION DE LITERATURA}

El bioabono es fácilmente obtenible, su contenido de nutrimentos es soluble en agua y puede ser aplicado en forma intensiva, Este material orgánico contiene nutrimentos suaves, de rápida eficacia y preservación prolongada. Se le considera como una fuente de reducción debido a que tiene una capacidad potencial de 50 a 150 milivoltios (mv), y poco menos de $200 \mathrm{mv}$. En estado fresco el bioabono perjudicaría la germinación y la vegetación, por lo que es necesario almacenarlo en piletas y cámaras de acumulación 3 a 5 días antes de usarlo (Instituto de Suelos y Fertilizantes, 1984).

El bioabono generalmente reduce la acidez del suelo, por un período prolongado de tiempo y lo enriquece químicamente (Oliveira et al, 1986). La reducción de la acidez del suelo es atribuida a la capacidad de bioabono de retener las hases para la formación de complejos orgánicos. Estas bases generalmente son transportadas a los horizontes inferiores por el agua de percolación (Black, 1975, Jenny y Leonard 1934, Citado por Oliveira et al, 1986).

Cuando se siembran cultivos sucesivos en la misma área aplicando bioabono antes de cada plantío, existe una tendencia de las dosis menores a equipararse, en producción con las mayores (Oliveira, et al, 1986).

En los sucios franco-arenosos a gruesos, el estiércol parece tener una influencia concreta sobre el equilibrio de la población y la actividad microbiana, lo cual tiene importantes consecuencias agronómicas (Charreau, 1974).

Experimentos de campo e invernadero parecen probar la hipótesis del desarrollo de una flora patógena cuando se agrega tílncamcnte estiércol al suelo. En cambio cuando existe una esterilización y agregación de estiércol los efectos son mejores, ya que con la primera los patógenos son destruidos y su degradación es acenluada por la adición de estiércol (Charreau, 1974).

\section{MATERIALES Y MÉTODOS}

El estudio de campo se estableció en la Escuela Nacional de Agricultura, Cantón San Andrés del Departamento de La Libertad a una altitud de $460 \mathrm{msnm}$, con una precipitación media anual de $1597 \mathrm{~mm}$ yuna temperatura media anual de 23.8
Se realizó durante dos años consecutivos 1988-1989 en la época lluviosa. El suelo en estudio fue un Regosol Aluvial de textura franco-arenosa.

Se evaluaron cinco tratamientos en un diseño de bloques al azar con cuatro repeticiones. En el cuadro 1 se muestran los tratamientos evaluados en los dos años.

El área total del ensayo fue de $475 \mathrm{~m}^{2}$, el área de la parcela experimental fue de $20 \mathrm{~m}^{2}(5 \times 4 \mathrm{~m})$ con un área útil de $11.20 \mathrm{~m}^{2}$.

Cuadro 1 Tratamientos evaluados y dósis aplicadas de fertilizantes 1988-1989.

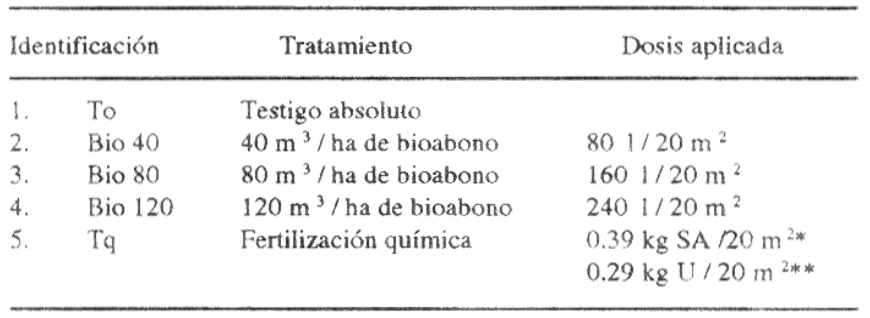

* SA $=$ Sulfato de amonio al $21 \%$ (1988).

** $\mathrm{U}=$ Urea al $46 \%$ (1989).

Como planta indicadora se utilizó la vigna (Vigna unguiculata var. Pin early eye). El distanciamiento de siembra fue de $60 \mathrm{~cm}$ entre surcos y $20 \mathrm{~cm}$ entre plantas, confonnando un total de 150 plantas por parcela experimental y 80 plantas por parcela útil (75,000 plantas/ha).

En 1988 inicialmente se aró el terreno con un arado de discos a una profundidad de $20 \mathrm{~cm}$, y posteriormente se realizaron dos pasos de rastra. Con el último paso de rastra se incorporó el insecticida al suelo (Counter 10\%) En cada año y ocho días antes de la siembra, se aplicaron al suelo las diferentes dosis de bioabono en estado fresco y luego fueron

Cuadro 2. Características químicas y físicas del bioabono aplicado.

\begin{tabular}{lrrr}
\hline & \% & kg & g/cc \\
\hline & & & \\
Humedad & 96.86 & & \\
Sólidos totales & 3.14 & & \\
Sólidos volátiles* & 58 & & 1.401 \\
Densidad & & & \\
$\mathrm{N}$ & & 0.886 & \\
$\mathrm{P}_{2} \mathrm{O}_{5}$ & & 1.34 & \\
$\mathrm{~K}_{2} \mathrm{O}$ & & 2.45 & \\
$\mathrm{Ca}$ & & 0.810 & \\
$\mathrm{Mg}$ & & 0.134 & \\
$\mathrm{Fe}$ & & 0.300 & \\
$\mathrm{pH}$ & & & \\
\hline
\end{tabular}

Análisis realizados en el Departamento de Química Agrícola del CENTA.

* Sólidos volátiles expresados como porcentaje del total de sólidos. 
incorporadas en forma manual a una profundidad de $15 \mathrm{~cm}$ En el cuadro 2 se presentan las características del bioabono aplicado. Al momento de la siembra se plantaron dos semillas por postura a una profundidad de 2 a $3 \mathrm{~cm}$.

En el tratamiento químico (Tq) se aplicaron ocho días después de la siembra, las dosis de sulfato de amonio y urea el primero y segundo año respectivamente. En ambos casos la aplicación fue en banda y luego incorporado manualmente. Se realizaron las prácticas culturales y aplicaciones de pesticidas necesarias para mantener el cultivo en condiciones óptimas de sanidad.

\section{Parámetros evaluados}

Porcentaje de germinación: Se tomó en toda la parcela experimental cinco días después de siembra, en base al recuento de las plantas germinadas y expfesado como porcentaje del total sembrado en la parcela.

Altura de plantas: El crecimiento ortotrópico se evaluó en dos épocas del cultivo, a los ocho días después de la siembra y a los treinta días después de la siembra.

Peso total de campo: Resultado de cinco pesos de campo correspondientes a los cinco cortes realizados en el cultivo. Las vainas fueron contadas y pesadas inmediatamente despues de cortadas.

Muestreo de suelo: Se realizó antes de la primera siem bra y después de la segunda cosecha con el fin de determinar las condiciones, y el contenido de nutrimentos y materia orgánica.

Análisis económico: Inicialmente se calculó el costo por metro cúbico de bioabono, tomando en cuenta los costos totales por año del biodigestor de donde se obtuvo. Dichos costos se relacionaron con el rendimiento del bioabono obtenido, resultando un costo de Colones $6.80 \mathrm{~m}^{3}$. (US\$ $100 / \mathrm{m}^{3}$ ). Luego en cada tratamiento se calculó su costo de producción agregando en los costos de mano de obra e in sumos el valor resultante de las diferentes aplicaciones de los fertilizantes usados.

\section{RESULTADOS Y DISCUSION}

En el cuadro 3 se muestran los rendimientos de la Vigna en peso fresco obtenidos en los dos años de aplicación de bioabono. En 1988 el mayor rendimiento promedio se obtuvo en el testigo absoluto ( $3.98 \mathrm{t} / \mathrm{ha}$ ) seguido del tratamiento con dosis de $80 \mathrm{~m}^{3} /$ ha de bioabono (3.75 t / ha), sin embargo el análisis de varianza no mostró diferencias significativas entre las medias de rendimiento para este período.

En 1989 el mayor rendimiento promedio se obtuvo con la fertilización química (4.36 t / ha) seguido del tratamiento
Cuadro 3. Rendimiento obtenido por tratamiento en dos años del cultivo de vigna, var. Pink early eye, 1988-1989.

\begin{tabular}{lcc}
\hline Tratamiento & \multicolumn{2}{c}{ Rendimiento de vigna en peso fresco ( $/$ / ha) } \\
\cline { 2 - 3 } & 1988 & 1989 \\
\hline 1. Fertilización & & \\
Química & 3.60 & 4.36 \\
2. Bio 40 & 3.68 & 4.04 \\
3. Bio 80 & 3.75 & 4.11 \\
4. Bio 120 & 3.58 & 4.17 \\
5. Testigo absoluto & 3.98 & 3.74 \\
& 3.72 & 4.08 \\
Medias & & \\
CV (\%) & 8.89 & 9.21 \\
\hline
\end{tabular}

con la dosis de $120 \mathrm{~m}^{3} /$ ha de bioabono (4.17 t/ha). Todos los tratamientos en donde fue aplicado el bioabono mostraron leves incrementos con respecto al año anterior aún cuando el anál isis de varianza tampoco indicó diferencias significativas entre las medias de rendimiento en este período.

Este leve incremento se debe probablemente al efecto acumulativo del bioabono en el suelo aunque no a los niveles reportados por Oliveira et al., (1986), en donde los resultados que se obtuvieron cuando se aplicó el bioabono en un suelo cultivado con frijol y posteriormente arroz indicaron dicho efecto.

Una razón que vuelve insensible el efecto de las aplicaciones de bioabono en este estudio es la fertilidad natural del suelo, ya que se encuentra en condiciones adecuadas, previéndose que a medida que transcurra el tiempo y el sucio se cultive más, dicha fertilidad decrecerá.

Tanto con las aplicaciones de bioabono y fertilizantes químicos se obtuvieron rendimentos similares a los obtenidos en la explotación comercial de la vigna, var. Pink early eye. Estudios similares en las mismas parcelas experimentales, evaluando el rendimiento del tomate y el repollo mostraron resultados similares como se observa en el cuadro 4.

Cuadro 4. Efecto del bioabono sobre el rendimiento de tomate y repollo, (t/ha)

\begin{tabular}{lccc}
\hline Tratamiento & \multicolumn{2}{c}{ Tomate } & Repollo \\
\cline { 2 - 3 } & Epoca lluviosa & Epoca seca & Epoca seca \\
\hline 1. Fertilización química & 13.5 & 12.9 & 47.4 \\
2. Bio 40 & 11.5 & 14.3 & 34.8 \\
3. Bio 80 & 13.0 & 17.0 & 36.4 \\
4. Bio 120 & 11.0 & 20.2 & 43.1 \\
5. Testigo absoluto & 12.4 & 9.6 & 39.4 \\
Medias & 12.28 & 14.80 & 40.22 \\
CV $(\%)$ & 6.95 & 8.75 & 10.03 \\
\hline
\end{tabular}


Cuadro 5. Características químicas del suelo antes y después de dos aplicaciones de bioabono.

\begin{tabular}{|c|c|c|c|c|c|c|c|c|c|c|c|c|c|c|c|c|}
\hline \multirow[t]{2}{*}{ Trat. } & \multicolumn{2}{|c|}{$\begin{array}{c}\text { pH en } \\
\text { agua 1:25 }\end{array}$} & \multicolumn{2}{|c|}{$\begin{array}{c}\mathrm{P} \\
\mathrm{ppm}\end{array}$} & \multicolumn{2}{|c|}{$\begin{array}{c}\mathrm{Ca}+\mathrm{Mg} \\
(\mathrm{meq} / 100 \mathrm{~g})\end{array}$} & \multicolumn{2}{|c|}{$\begin{array}{c}\mathrm{Na} \\
(\mathrm{meq} / 100 \mathrm{~g})\end{array}$} & \multicolumn{2}{|c|}{$\begin{array}{c}\mathrm{K} \\
(\mathrm{meq} / 100 \mathrm{~g})\end{array}$} & \multicolumn{2}{|c|}{$\begin{array}{c}\text { Bases } \\
\text { (meq } / 100 \mathrm{~g})\end{array}$} & \multicolumn{2}{|c|}{$\begin{array}{c}\text { Satur. Bases } \\
\%\end{array}$} & \multicolumn{2}{|c|}{$\begin{array}{c}\text { CIC } \\
\text { (meq/100g) }\end{array}$} \\
\hline & $A_{1}$ & $\mathrm{D}_{1}$ & $\mathrm{~A}_{1}$ & $\mathrm{D}_{2}$ & $\mathrm{~A}_{1}$ & $\mathrm{D}_{2}$ & $\mathrm{~A}_{2}$ & $\mathrm{D}_{2}$ & $\mathrm{~A}_{2}$ & $\mathrm{D}_{2}$ & $\mathrm{~A}_{2}$ & $\mathrm{D}_{2}$ & $\mathrm{~A}_{2}$ & $\mathrm{D}_{2}$ & $\mathrm{~A}_{1}$ & $\mathrm{D}_{2}$ \\
\hline 1. To & 6.4 & 6.3 & 67.5 & 64.0 & 14.4 & 15.6 & 0.31 & 0.27 & 2.67 & 2.35 & 21.0 & 18.2 & 72.8 & 69.1 & 31.1 & 26.6 \\
\hline 2. Bio 40 & 6.4 & 6.5 & 52.3 & 47.8 & 13.2 & 14.8 & 0.28 & 0.36 & 1.93 & 2.30 & 19.4 & 16.9 & 71.9 & 69.8 & 28.7 & 24.4 \\
\hline 3. $\mathrm{Bio} 80$ & 6.4 & 6.4 & 48.5 & 62.8 & 13.1 & 16.7 & 0.28 & 0.26 & 1.88 & 2.16 & 18.7 & 19.2 & 69.7 & 71.2 & 31.4 & 27.1 \\
\hline 4. Bio 12 & 6.5 & 6.4 & 50.0 & 53.3 & 12.6 & 14.6 & 0.34 & 0.26 & 2.06 & 2.25 & 19.0 & 17.1 & 70.5 & 72.7 & 28.4 & 23.8 \\
\hline 5. $\mathrm{Tq}$ & 6.4 & 6.5 & 54.8 & 57.0 & 13.3 & 16.0 & 0.40 & 0.25 & 1.60 & 2.64 & 18.1 & 18.9 & 71.3 & 83.1 & 32.8 & 23.2 \\
\hline
\end{tabular}

$A_{1}=$ Inicio, antes de primera aplicación y siembra

$A_{2}=$ Después de primera aplicación y cosecha

$\mathrm{D}_{2}=$ Después de segunda aplicación y dos cosechas

Cuadro 6. Efecto de la aplicación del bioabono sobre el contenido de hierro del suelo.

\begin{tabular}{lll}
\hline Tratamiento & \multicolumn{2}{c}{ Fe (ppm) } \\
\cline { 2 - 3 } & $\mathrm{A}_{1}$ & $\mathrm{D}_{2}$ \\
\hline Fertilización química & 26.1 & $48.5 \mathrm{a}$ \\
Bio 40 & 24.9 & $42.1 \mathrm{ab}$ \\
Bio 80 & 25.8 & $48.6 \mathrm{a}$ \\
Bio 120 & 24.6 & $52.5 \mathrm{a}$ \\
Testigo absoluto & 18.0 & $37.0 \mathrm{~b}$ \\
\end{tabular}

$A_{1}=$ Antes de siembra y aplicación abono

$\mathrm{D}_{2}=$ Después de tres cultivos + dos aplicaciones de abono

Las medias con la misma letra no difieren estadísticamente a un nivel del $5 \%$ según la prueba de Dunca

En cuanto a las propiedades químicas del suelo evaluadas en el cuadro 5 se muestran los resultados obtenidos al inicio y después de tres cultivos más dos ciclos de aplicación de fertilizantes.

En las parcelas que recibieron bioabono, se observaron leves incrementos en el contenido de fósforo $(\mathrm{P})$, calcio $(\mathrm{Ca})$ y magnesio $(\mathrm{Mg})$ y potasio $(\mathrm{K})$ interc:unbiable. Esto hace suponer que aplicaciones frecuentes de bioabono mantendrán o incrementarán los contenidos de estos elementos y de otros como el sodio $(\mathrm{Na})$ en el suelo.

El pH del suelo no varió en ninguno de los tratamientos manteniéndose casi estable en el rango de ligeramente ácido.

Los incrementos en los niveles de hierro $(\mathrm{Fe})$ que significativos con respecto al testigo absoluto, a medida la dosis de bioabono fue mayor. Esto puede deberse a la naturaleza misma del estiércol bovino que presenta altas concentraciones de este elemento. Dicha situación limitaría el uso del bioabono en suelos con altas proporciones de Fe (Cuadro 6).

En general las características químicas del suelo no han sido afectadas severamente durante el tiempo que tiene de desarrollo el estudio.
Cuadro 7. Utilidad neta obtenida en las diferentes aplicaciones de fertilizantes en el cultivo de vigna.

\begin{tabular}{lrrr}
\hline Tratamiento & \multicolumn{2}{c}{ Utilidad Neta (C./ha) } & \multirow{2}{*}{ Total } \\
\cline { 2 - 3 } & \multicolumn{1}{c}{1988} & 1989 & \\
\hline 1. Fertilización química & 1009.37 & 1525.87 & 2535.24 \\
2. Bio 40 & 1128.08 & 1446.03 & 2574.11 \\
3. Bio 80 & 894.89 & 1259.96 & 2154.85 \\
4. Bio 120 & 544.80 & 1078.01 & 1622.81 \\
5. Testigo absoluto & 1594.94 & 1337.00 & 2931.94 \\
\hline
\end{tabular}

Valores obtenidos en el período 1988-1989..

US $\$ 100=$ Colones 6.90

En el cuadro 7 se muestra la utilidad neta obtenida con las aplicaciones de bioabono y fertilización química. En el primer año la dosis de $40 \mathrm{~m}^{3} /$ ha de bioabono fue la que mostró una mayor utilidad neta por hectárea, Colones 1128.08 (US\$ 163.50) a mayor dosis aplicada de bioabono esta utilidad neta se vio reducida. Es posible por consiguiente que menores retornos pueden ser obtenidos si se incrementan las dosis de bioabono.

En el segundo año se observa que de las tres dosis aplicadas de bioabono la de $40 \mathrm{~m}^{3} / \mathrm{ha}$, nuevamente presenta la mayor utilidad neta Colones 1446.03 (US\$ 210.(0) siendo casi similar a la obtenida con la fertilización química. Sumándose tanto la utilidad del primer año como la del segundo, los resultados de la dosis de $40 \mathrm{~m}^{3} /$ ha de bioabono son bastante aproximados a los obtenidos con la fertilización convencional.

Lo anterior evidencia que la utilización del bioabono representa ganancias económicas. Aunque debe tomarse en cuenta que el uso de este material orgánico se ve limitado a superficies de 4.0 hectáreas como máximo y no deben estar alejadas de los biodigestores para no incurrir en un costo mayor por el traslado del bioabono. 


\section{CONCLUSIONES}

1. Los rendimientos de vigna en peso fresco fueron similares en los tratamientos con bioabono y la aplicación de fertilizantes químicos en ambos casos se obtuvieron rendimientos iguales a los obtenidos en forma comercial. Iguales resultados han sido obtenidos en estudios con los cultivos de tomate y repollo.

2. Se observó una leve tendencia creciente en los rendimientos de vigna obtenidos en este segundo año con respecto a los obtenidos en el primer año, lo que hace suponer que los efectos del bioabono sobre el rendimiento serán mejores a través del tiempo.

3. A diferencia del hierro, los demás parámetros químicos evaluados en el suelo no fueron afectados significativamente en ninguno de los tratamientos estudiados.

4. Dosis alrededor de $40 \mathrm{~m}^{3} / \mathrm{ha}$ de bioabono proporcionan buen retorno económ.ico en la producción de vigna;nin- gún problema ha sido observado hasta el momento en el aspecto vegetativo del cultivo con dosis de 80 a $120 \mathrm{~m}^{3} / \mathrm{ha}$.

\section{BIBLIOGRAFIA}

Charreu, C. 1974. Materia orgánica y propiedades bioquímicas del suelo en la zona tropical árida del Africa Occidental. In Organización de las Naciones Unidas para la Agricultura y la Alimentación. Boletín sobre Suelos, Roma N. 27:148-167.

Oliveira. L.P. et al. 1986. Resultados técnicos e económicos da aplicacao de biofertilizante bobina nas culturas de Feijao, arroz e trigo. Circular técnica (Brazil) N. 21:24.

INSTITUTO DE SUELOS Y FERTILIZANTES (República Popular China) 1984. El biogas en la Agricultura. In Organización de las Naciones Unidas para la Agricultura y la Alimentación; curso de capacitación sobre reciclaje de materias orgánicas y biogas; una experiencia en China, Santiago de Chile. 22 p. 